\title{
Cottage cheeses functionalized with fennel and chamomile extracts: Comparative performance between free and microencapsulated forms
}

\author{
Cristina Caleja $^{\mathrm{a}, \mathrm{b}, \mathrm{c}}$, Andreia Ribeiro ${ }^{\mathrm{a}, \mathrm{b}}$, Lillian Barros ${ }^{\mathrm{a}}$, João C.M. Barreira ${ }^{\mathrm{a}, \mathrm{c}}$, Amilcar L. Antonio $^{\mathrm{a}}$, \\ M. Beatriz P.P. Oliveira ${ }^{c}$, Maria Filomena Barreiro ${ }^{\mathrm{b}, *}$, Isabel C.F.R. Ferreira ${ }^{\mathrm{a}, *}$ \\ a Mountain Research Centre (CIMO), ESA, Polytechnic Institute of Bragança, Campus de Santa Apolónia, 1172, $5301-855$ Bragança, Portugal \\ ${ }^{\mathrm{b}}$ Laboratory of Separation and Reaction Engineering (LSRE), Associate Laboratory LSRE/LCM, IPB, Campus de Santa Apolónia, 1134, 5301-857 Bragança, Portugal \\ ${ }^{\mathrm{c}}$ REQUIMTE - Science Chemical Department, Faculty of Pharmacy, University of Porto, Rua Jorge Viterbo Ferreira $n^{\circ} 228,4050-313$ Porto, Portugal
}

\section{A R T I C L E I N F O}

\section{Article history:}

Received 10 September 2015

Received in revised form 10 November 2015

Accepted 15 December 2015

Available online 21 December 2015

\section{Keywords:}

Foeniculum vulgare

Matricaria recutita

Cottage cheese

Functional foods

Microencapsulation

\begin{abstract}
A B S T R A C T
Globally, there is a trend for healthy food products, preferably incorporating natural bioactive ingredients, replacing synthetic additives. From previous screening studies, extracts of Foeniculum vulgare Mill. (fennel) and Matricaria recutita L. (chamomile) maintained nutritional properties and improved the antioxidant activity of cottage cheese. Nevertheless, this effect was limited to 7 days. Accordingly, aqueous extracts of these plants were microencapsulated in alginate and incorporated into cottage cheese to achieve an extended bioactivity. Plain cottage cheese, and cheese functionalized by direct addition of free decoctions, were prepared and compared. Independently of plant species, "functionalization type" factor did not show a significant effect on the nutritional parameters, as also confirmed in the linear discriminant analysis, where these parameters were not selected as discriminating variables. Furthermore, samples functionalized with microencapsulated extracts showed higher antioxidant activity after the 7th day, thereby demonstrating that the main purpose of this experimental work was achieved.
\end{abstract}

(c) 2015 Elsevier Ltd. All rights reserved.

\section{Introduction}

Plant-derived bioactive extracts and compounds are interesting ingredients used to functionalize foods (Carocho, Barreiro, Morales, \& Ferreira, 2014). Aqueous extracts of Foeniculum vulgare Mill. (fennel) and Matricaria recutita L. (chamomile) are good sources of phenolic compounds, exhibiting different biological activities such as antioxidant and antimicrobial properties, as previously reported by our research group (Caleja, Barros, Antonio, Ciric, Barreira et al., 2015; Caleja, Barros, Antonio, Ciric, Soković et al., 2015). In these previous works, the preserving potential of fennel and chamomile extracts obtained from decoction was explored through their direct use as natural preservers. Their incorporation into cottage cheese maintained its nutritional characteristics and improved the antioxidant properties, namely the free radical's scavenging activity. However, after 7 days under storage the cheese samples showed an antioxidant capacity decrease which was associated with extract degradation (Caleja, Barros, Antonio, Ciric, Barreira et al., 2015; Caleja, Barros, Antonio, Ciric, Soković et al., 2015).

\footnotetext{
* Corresponding authors.

E-mail addresses: barreiro@ipb.pt (M.F. Barreiro), iferreira@ipb.pt
} (I.C.F.R. Ferreira).
In fact, the use of natural bioactive extracts/compounds as food additives presents limitations because after extraction they can become susceptible to degradation. Therefore, microencapsulation may be considered as an appropriate process to overcome these limitations, since this technique can provide protection against the action of several environmental agents like oxygen, light, moisture or heat, ensuring an increase in their stability (Betz \& Kulozik, 2011; Dias, Ferreira, \& Barreiro, 2015). This process will preserve the bioactive compound by means of a surrounding coating shell around it (reservoir type particles) or by embedding it, homogeneously or heterogeneously, in a matrix (matrix type particles) (Çam, Içyer, \& Erdogan, 2014). The controlled release along time or oriented to a specific site, can be achieved by means of different mechanisms, which depend on the used encapsulation materials, production process, and microcapsules' morphology and desired application (Martins et al., 2014). Alginate, a natural polymer obtained from bacteria and algae, is widely used for microencapsulation in several fields, namely in food industry (Goh, Heng, \& Chan, 2012). This polymer is classified as non-toxic for oral administration, and is usually commercialized in its salt form (e.g. sodium alginate). In the presence of bivalent cations (e.g. $\mathrm{Ca}^{2+}$ ) it gels, giving rise to a material that resists acidic $\mathrm{pH}$ and dissolves at basic medium (disruption of the ionic network). In this way the release of the encapsulated compounds will occur in the intestinal tract 
(George \& Abraham, 2006). Besides this, it presents good stability, biocompatibility, exudate-retaining ability and moderate antimicrobial activity (Goh et al., 2012). Its use in the food industry is permitted by the FDA - Food and Drug Administration (USA) and EFSA - European Food Safety Agency.

There are some documented examples dealing with the application of microencapsulation to natural extracts for use in functional foods (Dias, Ferreira et al., 2015). Our research group has successfully encapsulated Fragaria vesca L. (Dias, Barros et al., 2015) and Rubus ulmifolius Schott (Martins et al., 2014) extracts that were further incorporated into $\kappa$-carrageenan gelatin and yogurts, respectively.

In the present study, aqueous extracts of $F$. vulgare and $M$. recutita were prepared by decoction. Then, these extracts were used to functionalize cottage cheeses following two main strategies: (i) direct use (extracts in their free form), and (ii) use after stabilization through microencapsulation with alginate (extracts in their microencapsulated form). Microencapsulation was achieved by using an atomization/coagulation technique following a procedure developed in our research group (Dias, Barros et al., 2015; Martins et al., 2014). The incorporation of $F$. vulgare and $M$. recutita extracts into cottage cheeses was compared with samples without free or encapsulated extracts (control). Moreover the gain derived from the use of the microencapsulated form over the use of the free form was also evaluated (specifically color, nutritional value and antioxidant activity of the functionalized cottage cheese as a function of storage time). Besides studying individual changes induced by each of the defined factors (storage time and functionalization type) through a 2-way ANOVA, data were also analyzed by a linear discriminant analysis to determine which of the assayed independent variables (studied parameters) defined the majority of the differences in the average score profiles of the prepared cheese samples.

\section{Materials and methods}

\subsection{Standards and reagents}

2,2-Diphenyl-1-picrylhydrazyl (DPPH) was obtained from Alfa Aesar (Ward Hill, MA, USA). HPLC-grade acetonitrile was obtained from Merck KgaA (Darmstadt, Germany). Formic and acetic acids were purchased from Prolabo (VWR International, France). Sodium alginate was provided by Fluka Chemie (USA). All other chemicals and solvents were of analytical grade and purchased from common sources. Water was treated in a Milli-Q water purification system (TGI Pure Water Systems, Greenville, SC, USA).

\subsection{Preparation of the bioactive extracts}

Commercial samples of $F$. vulgare Mill. (fennel) and M. recutita L. (chamomile) were provided by Américo Duarte Paixão Lda. (Alcanede, Portugal). The dried samples were powdered ( $\sim 20$ mesh) and submitted to decoction. Decoctions were performed by adding $5 \mathrm{~g}$ of plant material to $200 \mathrm{~mL}$ of distilled water, heated (heating plate, VELP scientific, Usmate, Italy), and allowed to boil for $5 \mathrm{~min}$. The mixtures were left to stand for $5 \mathrm{~min}$ and filtered through Whatman No. 4 paper. The decoctions were then frozen and lyophilized in order to obtain the final extracts (FreeZone 4.5, Labconco, Kansas City, MO, USA).

\subsection{Microencapsulation of the plant extracts and characterization}

\subsubsection{Microencapsulation}

Microspheres containing extracts of $F$. vulgare or $M$. recutita were prepared by using an atomization/coagulation technique as previously described in the literature (Dias, Barros et al., 2015; Martins et al., 2014).
Calcium alginate (matrix material) was obtained by combining sodium alginate with calcium chloride $\left(\mathrm{CaCl}_{2}\right)$ (coagulation agent). Briefly, the atomization solution was prepared by dissolving firstly, $100 \mathrm{mg}$ of the extract with $20 \mathrm{~mL}$ of distilled water under stirring at $250 \mathrm{rpm}$ and room temperature, followed by filtration to remove remaining non-soluble trace residues. In the second step, $800 \mathrm{mg}$ of sodium alginate was added and the solution was kept stirring, under the same conditions, until complete dissolution was achieved. The obtained alginate solution containing the extract was then atomized using a NISCO Var J30 system (Zurich, Switzerland) at a feed rate of $0.2 \mathrm{~mL} / \mathrm{min}$ and a nitrogen pressure of 0.1 bar to produce the microspheres. The atomized microspheres underwent coagulation upon contact with a $\mathrm{CaCl}_{2}$ aqueous solution ( $500 \mathrm{~mL}$ at a concentration of $4 \%(\mathrm{w} / \mathrm{v})$ ) over a period of $4 \mathrm{~h}$. The resulting microspheres were collected by filtration under reduced pressure, washed twice with distilled water, and further lyophilized and stored in the dark at $4{ }^{\circ} \mathrm{C}$.

\subsubsection{Microcapsules characterization}

Microspheres were analyzed by optical microscopy (OM) using a Nikon Eclipse 50i microscope (Tokyo, Japan) equipped with a Nikon Digital Sight camera and NIS Elements software for data acquisition. OM analysis was applied to assess the size and morphology of the microspheres after the atomization and coagulation stages. It was also possible to infer the presence/absence of extract inside the microspheres.

The effective extract incorporation into the alginate matrix was investigated by FTIR analysis. For that purpose, spectra of pure alginate, free extracts of $F$. vulgare or $M$. recutita, and the corresponding microspheres were collected on a FTIR Bomen (model $\mathrm{MB} 104)$ by preparing $\mathrm{KBr}$ pellets at a sample concentration of $1 \%(\mathrm{w} / \mathrm{w})$. The spectra were recorded at a resolution of $4 \mathrm{~cm}^{-1}$ in the spectral range between 650 and $4000 \mathrm{~cm}^{-1}$ and by co-adding 48 scans. The encapsulation efficiency (EE) was also evaluated through the quantification of the non-encapsulated extract. For this purpose, the remaining extract in the coagulation and in the first washing solution were quantified by HPLC and added. The second washing solution presented no extract. The encapsulation efficiency was calculated according to the following expression:

$\mathrm{EE}=\left[\left(M_{\mathrm{e}-\mathrm{t}}-M_{\mathrm{e}-\mathrm{ne}}\right)\left(M_{\mathrm{e}-\mathrm{t}}\right)\right] \times 100$

in which $M_{\mathrm{e}-\mathrm{t}}$ represents the theoretical amount of extract (the amount of extract used in the microencapsulation process), $M_{\mathrm{e}-\mathrm{ne}}$ corresponds to the non-encapsulated extract remaining after the encapsulation process.

Since the extracts are complex mixtures, only the major phenolic compounds present in the extracts of fennel (quercetin-3-Oglucoside; Caleja, Barros, Antonio, Ciric, Soković et al., 2015) and chamomile (luteolin-O-glucuronide; Caleja, Barros, Antonio, Ciric, Barreira et al., 2015) were selected for EE evaluation.

\subsection{Functionalization of cottage cheese with plant extracts}

\subsubsection{Preparation of the cottage cheese samples}

All of the cottage cheese samples were prepared by "Queijos Casa Matias Lda." (Seia, Portugal), by using the milk serum obtained after the production of cheese. The remaining serum (liquid component) was pumped into a vat where it was mixed and heated to a temperature that ranged between 83 and $85^{\circ} \mathrm{C}$. After a few minutes at that temperature, the serum started to flocculate and rose to the surface where it was scooped into individual forms, left for a few minutes and packed with parchment paper. The incorporation of the extracts was carried out immediately before packaging, individually, into each one of the forms mentioned above, in order to guarantee a better distribution of the extract by the cottage cheese mass. 
Five groups, each comprising nine ewe's milk cottage cheeses $(250$ g), were prepared: (i) cottage cheeses without plant extracts; (ii) cottage cheeses with free fennel extract; (iii) cottage cheeses with free chamomile extract; (iv) cottage cheeses with microencapsulated fennel extract and (v) cottage cheeses with chamomile microencapsulated extract. For the samples prepared with free extracts, $100 \mathrm{mg}$ of extract per cottage cheese $(250 \mathrm{~g})$ were used. In the case of microencapsulated extract, $900 \mathrm{mg}$ of microspheres (quantity containing $100 \mathrm{mg}$ of free extract) per cottage cheese were used. The samples were analyzed according to color, nutritional composition (protein, fat, carbohydrates, ash and energy) and free radical scavenging activity, immediately after preparation, and also after seven, and fourteen, days of storage in the original package (parchment paper) at $4{ }^{\circ} \mathrm{C}$ in the refrigerator. Assays were carried out in triplicate.

\subsubsection{Effects of storage time on color, nutritional and antioxidant activity of cottage cheese samples}

The color of the sampled cottage cheeses was evaluated in the top and bottom of the cheese, by taking readings at three different points for each determination, using a colorimeter (model CR-400, Konica Minolta Sensing Inc., Tokyo, Japan). Using the illuminant C and a diaphragm aperture of $8 \mathrm{~mm}$, the CIE $L^{*} a^{*} b^{*}$ color space values were registered using the data software "Spectra Magic Nx" (version CM-S100W 2.03.0006) (Fernandes et al., 2012).

The proximate composition (protein, fat and ash) of the samples was analyzed according to the AOAC (2005) procedures.

The crude protein content $(N \times 6.38)$ was estimated by the Kjeldahl method (AOAC, 991.02); the crude fat (AOAC, 989.05) was determined by extracting a known weight of powdered sample with petroleum ether, using a Soxhlet apparatus; the ash content (AOAC, 935.42) was determined by incineration at $600 \pm 15^{\circ} \mathrm{C}$. Total carbohydrates were calculated by difference. Total energy was calculated according to the following equation: Energy $(\mathrm{kcal})=4 \times($ g proteins $+\mathrm{g}$ carbohydrates $)+9 \times($ g lipids $) . \quad$ Free sugars were determined in defatted samples by HPLC coupled to a refraction index (RI) detector, according to previous analytical validation and detailed description (Barros et al., 2013). Fatty acids were identified by comparison with standards and the results were expressed in relative percentage of each fatty acid. Free sugars were identified by comparison with standards, and further quantified ( $\mathrm{g} / 100 \mathrm{~g}$ of cottage cheese) by using an internal standard (melezitose).

For the antioxidant activity evaluation, the samples were submitted to DPPH radical scavenging activity, which was performed in an ELX800 microplate reader (Bio-Tek Instruments, Inc., Winooski, VT, USA) at $515 \mathrm{~nm}$. The complete protocol has previously been described by the authors (Caleja, Barros, Antonio, Ciric, Soković et al., 2015).

\subsection{Statistical analysis}

All statistical tests were performed at a $5 \%$ significance level using IBM SPSS Statistics for Windows, version 22.0. (IBM Corp., Armonk, NY, USA).

Data were expressed as mean \pm standard deviation, maintaining the significant numbers allowed by the magnitude of the standard deviation. An analysis of variance (ANOVA) with type III sums of squares was performed using the general linear model (GLM) procedure. The dependent variables were analyzed using 2-way ANOVA with the factors "storage time" (ST) and "functionalization type" (FT). When a statistically significant interaction was detected for these two factors, they were evaluated simultaneously by the estimated marginal means plots for all levels of each factor. Furthermore, if no statistical significant interaction was found, the means were compared using Tukey's multiple comparison test, with a previous assessment of the equality of variances through a Levene's test.

In addition, a linear discriminant analysis (LDA) was used to compare the effect of ST and FT over all the assayed parameters. A stepwise technique was applied, considering the Wilks' $\lambda$ test with the usual probabilities of $F$ (3.84 to enter and 2.71 to be removed) for variable selection. This procedure uses a combination of forward selection and backward elimination steps, where the inclusion of a new variable is preceded by verifying the significance of all previously selected variables (Zielinski et al., 2014). The basic purpose of the discriminant analysis was to estimate the relationship between a single categorical dependent variable (cheese formulation) and a set of quantitative independent variables (the values obtained in all of the assays). Through this method, it is possible to determine which of the independent variables contributed the most to the differences in the average score profiles of the different cheese samples. To verify the significance of the canonical discriminating functions, Wilk's $\lambda$ test was used. A leave-one-out cross validation procedure was carried out to assess the model performance.

\section{Results and discussion}

\subsection{Characterization of the microencapsulated plant extracts}

The OM analysis showed efficient incorporation of the extracts, confirming a homogeneous distribution of the extract within the microspheres, recognized as brown droplets well distributed in the alginate matrix. It was also observed that the microspheres had different shapes and sizes; the larger showed a round shape while the smaller had a pear-like shape. Their final size at a magnification of $100 \times$ varied between 68.1 and $306.5 \mu \mathrm{m}$. Fig. 1 shows the microcapsules morphology at different preparation stages, as well as after lyophilization. The EE, determined by quantification of the major compounds identified in the extracts (quercetin-3$O$-glucoside for microspheres with fennel and 5-O-caffeoylquinic acid for microspheres with chamomile), was estimated as approaching $100 \%$ for both samples. Only traces of these compounds were detected in the coagulation and washing solutions. The presence of the extract inside the microspheres was also confirmed by FTIR analysis (data not shown).

\subsection{Effects of incorporating plant-based natural extracts into cottage cheese}

Five groups of cottage cheese were prepared: (i) control (samples without plant extracts); (ii) samples with free fennel extract; (iii) samples with free chamomile extract; (vi) samples with microencapsulated fennel extract; and (v) samples with microencapsulated chamomile extract.

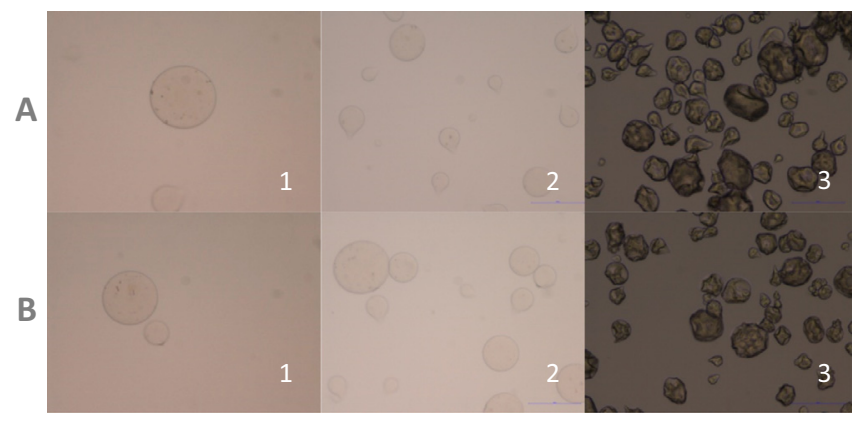

Fig. 1. Morphology of fennel (A) and chamomile (B) microspheres by OM analysis under $100 \times$ magnification, immediately after atomization (1), and after a $4 \mathrm{~h}$ coagulation period (2) and of lyophilized microspheres (3). 
For all of the evaluated parameters, the aim was to assess the effects of each functionalization type (i.e. functionalization agent used: free or microencapsulated extracts), independently of the number of days kept in storage, as well as the influence of storage time, regardless of whether the sample was plain cottage cheese or was functionalized. Therefore, the standard deviations should not be regarded as a measure of accuracy of the applied methodologies, since they reflect results obtained from samples prepared in different conditions. The interaction among factors $(\mathrm{ST} \times \mathrm{FT})$ was also evaluated. When a significant interaction was found $(p<0.050)$, no multiple comparisons were performed. In such cases, the influence of each factor was assessed by interpreting the estimated marginal mean (EMM) plots. The statistical analysis was performed separately for each of the selected plant species.

The achieved results for water, fat, protein, ash, carbohydrates, lactose and energy in the prepared cottage cheeses are presented in Table 1. The evaluated factors showed a significant interaction $(\mathrm{ST} \times \mathrm{FT})$ in all cases, indicating that the quantitative variations in these parameters evolved dissimilarly throughout ST, either using fennel or chamomile. Considering each individual factor, it is obvious that ST had a higher effect on these parameters than FT. In fact, while the variations induced by ST were significant in all cases, FT produced a significant effect only in ash (for fennel) and carbohydrates (for both plant species). Except for water, ash and lactose, which tended to decrease along ST, the assayed parameters tended to present higher values for longer storage periods (especially after 14 days). A similar behavior has previously been reported in cheeses of Portuguese (Caleja, Barros, Antonio, Ciric, Barreira et al., 2015), Spanish (Diezhandino, Fernández, González, McSweeney, \& Fresno, 2015) and Iranian (Farahani, Ezzatpanah, \& Abbasi, 2014) origin.

The interaction between factors had a less marked effect on the color parameters, since it only significantly impacted, in both plants, the $b^{*}$ parameter, thereby allowing to perform some multiple comparisons to be performed (Table 2). Lightness $\left(L^{*}\right)$ diminished along ST, also showing a tendency to be lower in functionalized samples (particularly in those with direct incorporation of the extracts). Despite the observed differences, the $L^{*}$ values were generally in the range of those obtained in similar cheese types (Caleja, Barros, Antonio, Ciric, Barreira et al., 2015; Pizzillo, Claps, Cifuni, Fedele, \& Rubino, 2005; Queiroga et al., 2013). Regarding the $b^{*}$ parameter, the highest values were obtained in samples stored for 14 days, exhibiting a yellowing tendency independently of FT. The increase in this particular parameter is a common feature in stored cheeses (Buffa, Trujillo, Pavia, \& Guamis, 2001; Ginzinger, Jaros, Lavanchy, \& Rohm, 1999; Rohm \& Jaros, 1996), which seems to be maintained in functionalized formulations, such as those prepared herein. For the $a^{*}$ parameter, which can be interpreted as a direct indicator of greenness-redness tendency, it was possible to observe a slight increase along ST, while the control and functionalized samples gave statistically indistinguishable results. However, the magnitude of the values obtained for this parameter is in the expected range for this type of product (Caleja, Barros, Antonio, Ciric, Barreira et al., 2015; Caleja, Barros, Antonio, Ciric, Soković et al., 2015; Pizzillo et al., 2005).

Fennel and chamomile have previously been reported to have high antioxidant activity, as assayed in alcoholic and aqueous extracts (Barros, Heleno, Carvalho, \& Ferreira, 2009; Guimarães et al., 2013; Mata et al., 2007). In previous studies, it was reported that the incorporation of chamomile or fennel improved the antioxidant activity of cottage cheese (Caleja, Barros, Antonio, Ciric, Barreira et al., 2015; Caleja, Barros, Antonio, Ciric, Soković et al., 2015). Herein, the incorporation of chamomile- and fennelbased ingredients improved the DPPH scavenging activity of plain cottage cheese samples, especially in the case of extracts, either using chamomile or fennel (Table 2). Since the compounds present in the extract were readily available when they were incorporated in the free form, it could intuitively be predicted that the antioxidant activity would be higher in these cheeses. However, it has previously been verified that the DPPH scavenging activity tends to decrease throughout the shelf-life of a functionalized cheese (Caleja, Barros, Antonio, Ciric, Barreira et al., 2015). Thereby, the encapsulation in microspheres could be hypothesized to preserve the scavenging activity throughout the shelf-life of the developed product. As can be seen in Fig. 2, samples functionalized with free extracts tended to lose some of the initial activity; on the other hand, it is obvious (particularly after the 7th day of storage) that the microencapsulation of the extracts promotes increasing levels of scavenging activity throughout ST, which represents a good indicator of the protective effect intended with the application of the microencapsulation technique.

The significant interaction between factors was also observed for all of the fatty acids studied (FA), with most of them also being significantly changed by each individual factor (Table 3 ). Neverthe-

Table 1


deviation.

\begin{tabular}{|c|c|c|c|c|c|c|c|c|}
\hline & & Water & Fat & Protein & Ash & Carbohydrates & Lactose & Energy \\
\hline \multicolumn{9}{|c|}{ Foeniculum vulgare } \\
\hline \multirow[t]{4}{*}{ ST } & 0 days & $70 \pm 1$ & $14 \pm 1$ & $12.0 \pm 0.3$ & $1.3 \pm 0.1$ & $2.5 \pm 0.4$ & $1.9 \pm 0.1$ & $186 \pm 7$ \\
\hline & 7 days & $66 \pm 1$ & $17 \pm 1$ & $12.4 \pm 0.2$ & $1.1 \pm 0.1$ & $3.6 \pm 0.4$ & $1.1 \pm 0.4$ & $215 \pm 3$ \\
\hline & 14 days & $54 \pm 2$ & $24 \pm 1$ & $18.0 \pm 0.5$ & $1.0 \pm 0.1$ & $3.7 \pm 0.5$ & $0.2 \pm 0.1$ & $301 \pm 9$ \\
\hline & $p$-Value $(n=27)$ & $<0.001$ & $<0.001$ & $<0.001$ & $<0.001$ & 0.007 & $<0.001$ & $<0.001$ \\
\hline \multirow[t]{4}{*}{ FT } & None & $63 \pm 7$ & $18 \pm 4$ & $15 \pm 3$ & $1.1 \pm 0.1$ & $3.2 \pm 0.5$ & $1.3 \pm 0.4$ & $238 \pm 49$ \\
\hline & Microspheres & $64 \pm 6$ & $18 \pm 4$ & $14 \pm 2$ & $1.2 \pm 0.1$ & $2.9 \pm 0.5$ & $1.0 \pm 0.5$ & $229 \pm 46$ \\
\hline & Extract & $63 \pm 8$ & $18 \pm 4$ & $14 \pm 3$ & $1.1 \pm 0.1$ & $3.6 \pm 0.5$ & $0.9 \pm 0.5$ & $235 \pm 55$ \\
\hline & $p$-Value $(n=27)$ & 0.443 & 0.532 & 0.546 & $<0.001$ & $<0.001$ & 0.906 & 0.459 \\
\hline $\mathrm{ST} \times \mathrm{FT}$ & $p$-Value $(n=81)$ & $<0.001$ & $<0.001$ & $<0.001$ & 0.013 & $<0.001$ & $<0.001$ & $<0.001$ \\
\hline \multicolumn{9}{|c|}{ Matricaria recutita } \\
\hline \multirow[t]{4}{*}{ ST } & 0 days & $69 \pm 1$ & $15 \pm 1$ & $12 \pm 1$ & $1.2 \pm 0.1$ & $3 \pm 1$ & $1.8 \pm 0.1$ & $193 \pm 4$ \\
\hline & 7 days & $66 \pm 1$ & $17 \pm 1$ & $12 \pm 1$ & $1.1 \pm 0.1$ & $4 \pm 1$ & $1.6 \pm 0.1$ & $215 \pm 6$ \\
\hline & 14 days & $53 \pm 2$ & $24 \pm 1$ & $18 \pm 1$ & $1.1 \pm 0.1$ & $4 \pm 1$ & $0.2 \pm 0.1$ & $304 \pm 13$ \\
\hline & $p$-Value $(n=27)$ & $<0.001$ & $<0.001$ & $<0.001$ & $<0.001$ & $<0.001$ & $<0.001$ & $<0.001$ \\
\hline \multirow[t]{4}{*}{ FT } & None & $63 \pm 7$ & $19 \pm 4$ & $15 \pm 3$ & $1.1 \pm 0.1$ & $3 \pm 1$ & $1.3 \pm 0.5$ & $238 \pm 49$ \\
\hline & Microspheres & $64 \pm 6$ & $18 \pm 4$ & $14 \pm 3$ & $1.2 \pm 0.1$ & $3 \pm 1$ & $1.2 \pm 0.5$ & $228 \pm 44$ \\
\hline & Extract & $62 \pm 8$ & $19 \pm 4$ & $14 \pm 3$ & $1.1 \pm 0.1$ & $5 \pm 1$ & $1.2 \pm 0.5$ & $245 \pm 54$ \\
\hline & $p$-Value $(n=27)$ & 0.753 & 0.863 & 0.681 & 0.056 & 0.004 & 0.244 & 0.793 \\
\hline $\mathrm{ST} \times \mathrm{FT}$ & $p$-Value $(n=81)$ & $<0.001$ & $<0.001$ & $<0.001$ & $<0.001$ & $<0.001$ & $<0.001$ & $<0.001$ \\
\hline
\end{tabular}


Table 2

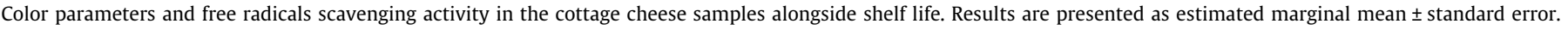

\begin{tabular}{|c|c|c|c|c|c|}
\hline & & DPPH scavenging activity & $L^{*}$ & $b^{*}$ & $a^{*}$ \\
\hline \multicolumn{6}{|c|}{ Foeniculum vulgare } \\
\hline \multirow[t]{4}{*}{ ST } & 0 days & $152 \pm 70$ & $91 \pm 3 a$ & $10 \pm 1$ & $-2.3 \pm 0.2 b$ \\
\hline & 7 days & $152 \pm 69$ & $90 \pm 4 \mathrm{ab}$ & $11 \pm 1$ & $-2.2 \pm 0.2 b$ \\
\hline & 14 days & $144 \pm 44$ & $89 \pm 2 b$ & $16 \pm 2$ & $-0.9 \pm 0.3 a$ \\
\hline & $p$-Value $(n=27)$ & 0.885 & 0.001 & $<0.001$ & $<0.001$ \\
\hline \multirow[t]{4}{*}{ FT } & None & $200^{*}$ & $92 \pm 2 \mathrm{a}$ & $12 \pm 3$ & $-2.0 \pm 0.4$ \\
\hline & Microspheres & $178 \pm 31$ & $91 \pm 3 a$ & $13 \pm 3$ & $-1.8 \pm 0.5$ \\
\hline & Extract & $69 \pm 20$ & $88 \pm 3 b$ & $12 \pm 2$ & $-1.7 \pm 0.5$ \\
\hline & $p$-Value $(n=27)$ & $<0.001$ & 0.003 & 0.054 & 0.256 \\
\hline $\mathrm{ST} \times \mathrm{FT}$ & $p$-Value $(n=81)$ & $<0.001$ & 0.052 & 0.005 & 0.263 \\
\hline \multicolumn{6}{|c|}{ Matricaria recutita } \\
\hline \multirow[t]{4}{*}{ ST } & 0 days & $145 \pm 80$ & $92 \pm 2 \mathrm{a}$ & $11 \pm 1$ & $-2.3 \pm 0.2$ \\
\hline & 7 days & $151 \pm 70$ & $91 \pm 2 \mathrm{a}$ & $11 \pm 1$ & $-2.3 \pm 0.4$ \\
\hline & 14 days & $142 \pm 60$ & $89 \pm 2 b$ & $17 \pm 2$ & $-0.8 \pm 0.2$ \\
\hline & $p$-Value $(n=27)$ & 0.856 & 0.009 & $<0.001$ & $<0.001$ \\
\hline \multirow[t]{4}{*}{ FT } & None & $200^{*}$ & $92 \pm 2 \mathrm{a}$ & $12 \pm 3$ & $-2.0 \pm 0.5$ \\
\hline & Microspheres & $188 \pm 19$ & $90 \pm 2 b$ & $13 \pm 3$ & $-1.6 \pm 0.5$ \\
\hline & Extract & $50 \pm 12$ & $90 \pm 2 b$ & $14 \pm 4$ & $-1.8 \pm 0.5$ \\
\hline & $p$-Value $(n=27)$ & $<0.001$ & $<0.001$ & 0.460 & 0.292 \\
\hline $\mathrm{ST} \times \mathrm{FT}$ & $p$-Value $(n=81)$ & $<0.001$ & 0.076 & $<0.001$ & 0.034 \\
\hline
\end{tabular}

Corresponds to the maximum assayed concentration. Different letters mean significant statistical differences.

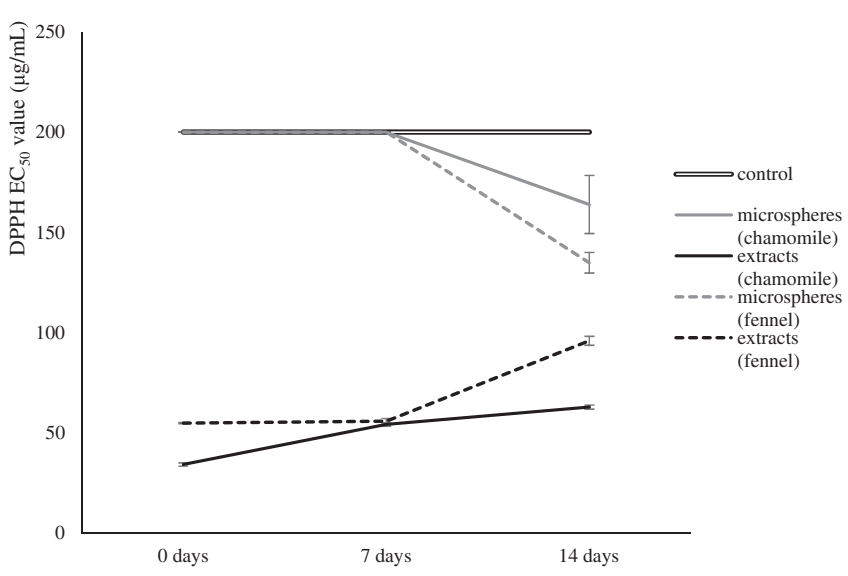

Fig. 2. Effects of storage time and functionalizing agent on the DPPH scavenging activity of cottage cheese functionalized with chamomile- or fennel-based extracts.

less, given the number of detected significant changes, the chamomile extracts seemed to have a higher capacity to maintain the FA profiles of plain cottage cheese. Besides the tabled fatty acids, C11:0, C13:0, C14:1, C15:0, C16:1, C17:0, C17:1, C18:2, C18:3, $\mathrm{C} 20: 1, \mathrm{C} 20: 4, \mathrm{C} 21: 0$ and C20:5 were also detected, but in trace amounts $(<0.2 \%)$. As verified in previous studies, saturated fatty acids (SFA) were the predominant forms, followed by monounsaturated (MUFA) and small amounts of polyunsaturated (PUFA), which is in agreement with the FA profiles typically detected in this type of lactic product (Caleja, Barros, Antonio, Ciric, Barreira et al., 2015; Caleja, Barros, Antonio, Ciric, Soković et al., 2015; Pizzillo et al., 2005; Queiroga et al., 2013).

\subsection{Linear discriminant analysis}

As discussed in Section 3.1, it was possible to identify different statistically significant effects (either induced by ST or FT) in most of the evaluated parameters. However, to characterize better each level ("0 days", "7 days" and "14 days" in one case, "none", "microspheres" and "extracts", in the other) of the assayed factors, it was essential to know which of the parameters contributed most decisively to the definition of those levels. Accordingly, different linear discriminant analysis (LDA) was performed with the basic objective of evaluating the linkage between the ST or FT levels (categorical dependent variables) and the matrix of obtained results (quantitative independent variables). The significant independent variables were selected following the stepwise method of the LDA, according to the Wilks' $\lambda$ test. Only variables with a statistically significant classification performance $(p<0.050)$ were maintained by the statistical model.

Initially, the results obtained for fennel were compared considering the effects induced by ST (Fig. 3a and b). The two defined discriminant functions included $100 \%$ of the observed variance by selecting fat, protein, lactose, energy, DPPH, $b^{*}, \mathrm{C} 4: 0,-\mathrm{C} 12: 0$, C14:0, C18:1 and PUFA as the variables with the strongest discriminant effect. Function 1, which was mostly correlated (data not shown) to fat, protein and energy (higher in "14 days" samples) projected the markers corresponding to the 14 day period far from the remaining samples, proving their significant differences. On the other hand, the separation of non-stored samples and those stored for 7 days was dictated by function 2, which was highly correlated to lactose (higher in non-stored samples), C12:0 and C14:0 (lower in non-stored samples).

Regarding the effect of FT, function 1, which separated markers corresponding to the extracts, was more correlated to DPPH (higher scavenging activity in "extracts") and lactose (higher in non-functionalized samples). Markers from non-functionalized samples and those incorporated with microencapsulated extracts scored differently regarding function 2 , which correlated more to SFA, and, particularly, with C18:0 (higher in non-functionalized samples). The other selected variables were water, fat, carbohydrates, $b^{*}$, C6:0, C10:0, C16:0 and PUFA.

A similar study was applied to the results obtained from chamomile-based functionalized cheese. The two defined functions regarding ST effect included $100.0 \%$ of the observed variance, selecting the variables water, protein, lactose, DPPH, C4:0, C10:0, C12:0, C16:0 and C18:0 as those with significant discriminant ability. The graph representation (Fig. 3c) indicates clearly that samples stored for 14 days are those with highest differences. The correlation among variables and discriminant functions specifies that lactose, water (clearly lower in "14 days" samples) and protein (higher in "14 days" samples) were the variables which contributed most to this separation. On the other hand, non-stored samples and those submitted to 7 days of storage were nearly equal regarding the variables correlated with function 1 ; their 
Table 3

Fatty acids profile (\%) of the cottage cheese samples alongside shelf life. Results are presented as estimated mean \pm standard deviation.

\begin{tabular}{|c|c|c|c|c|c|c|c|c|c|c|c|c|c|}
\hline & & $\mathrm{C} 4: 0$ & C6:0 & $\mathrm{C} 8: 0$ & C10:0 & $\mathrm{C} 12: 0$ & $\mathrm{C} 14: 0$ & C16:0 & C18:0 & C18:1 & SFA & MUFA & PUFA \\
\hline \multicolumn{14}{|c|}{ Foeniculum vulgare } \\
\hline \multirow[t]{4}{*}{ ST } & 0 days & $4.0 \pm 0.1$ & $3.5 \pm 0.1$ & $3.3 \pm 0.1$ & $8.1 \pm 0.2$ & $4.7 \pm 0.1$ & $10.7 \pm 0.2$ & $23.7 \pm 0.3$ & $12 \pm 2$ & $22 \pm 2$ & $72 \pm 2$ & $24 \pm 2$ & $3.8 \pm 0.1$ \\
\hline & 7 days & $3.7 \pm 0.3$ & $3.8 \pm 0.1$ & $3.6 \pm 0.1$ & $8.8 \pm 0.2$ & $5.0 \pm 0.1$ & $11.1 \pm 0.3$ & $24.1 \pm 0.5$ & $12 \pm 2$ & $20 \pm 1$ & $74 \pm 1$ & $22 \pm 1$ & $3.6 \pm 0.2$ \\
\hline & 14 days & $2.4 \pm 0.5$ & $3.2 \pm 0.4$ & $3.4 \pm 0.2$ & $8.5 \pm 0.3$ & $5.0 \pm 0.2$ & $11.1 \pm 0.2$ & $24.1 \pm 0.3$ & $13 \pm 2$ & $21 \pm 2$ & $73 \pm 2$ & $23 \pm 2$ & $3.9 \pm 0.3$ \\
\hline & $p$-Value $(n=27)$ & $<0.001$ & $<0.001$ & $<0.001$ & $<0.001$ & $<0.001$ & $<0.001$ & 0.001 & 0.053 & 0.002 & $<0.001$ & $<0.001$ & $<0.001$ \\
\hline \multirow[t]{4}{*}{ FT } & None & $3.5 \pm 0.4$ & $3.5 \pm 0.1$ & $3.3 \pm 0.1$ & $8.3 \pm 0.3$ & $4.9 \pm 0.2$ & $10.9 \pm 0.3$ & $24.0 \pm 0.4$ & $14 \pm 1$ & $19 \pm 1$ & $75 \pm 1$ & $21 \pm 1$ & $3.8 \pm 0.1$ \\
\hline & Microspheres & $3.7 \pm 0.5$ & $3.7 \pm 0.2$ & $3.5 \pm 0.2$ & $8.6 \pm 0.4$ & $4.9 \pm 0.1$ & $11.1 \pm 0.2$ & $24.3 \pm 0.2$ & $10 \pm 1$ & $22 \pm 1$ & $72 \pm 2$ & $25 \pm 2$ & $3.8 \pm 0.1$ \\
\hline & Extract & $3.0 \pm 0.5$ & $3.3 \pm 0.4$ & $3.4 \pm 0.2$ & $8.5 \pm 0.3$ & $4.9 \pm 0.2$ & $10.8 \pm 0.2$ & $23.7 \pm 0.4$ & $13 \pm 1$ & $21 \pm 1$ & $73 \pm 1$ & $23 \pm 1$ & $3.8 \pm 0.4$ \\
\hline & $p$-Value $(n=27)$ & 0.015 & $<0.001$ & $<0.001$ & 0.005 & 0.328 & 0.006 & $<0.001$ & $<0.001$ & $<0.001$ & $<0.001$ & $<0.001$ & 0.443 \\
\hline $\mathrm{ST} \times \mathrm{FT}$ & $p$-Value $(n=81)$ & $<0.001$ & 0.001 & 0.001 & $<0.001$ & $<0.001$ & $<0.001$ & $<0.001$ & $<0.001$ & $<0.001$ & $<0.001$ & $<0.001$ & $<0.001$ \\
\hline \multicolumn{14}{|c|}{ Matricaria recutita } \\
\hline \multirow[t]{4}{*}{ ST } & 0 days & $3.9 \pm 0.1$ & $3.5 \pm 0.1$ & $3.3 \pm 0.1$ & $8.2 \pm 0.2$ & $4.7 \pm 0.1$ & $10.7 \pm 0.2$ & $23.7 \pm 0.3$ & $12 \pm 2$ & $22 \pm 1$ & $73 \pm 1$ & $24 \pm 1$ & $3.7 \pm 0.1$ \\
\hline & 7 days & $3.8 \pm 0.3$ & $3.7 \pm 0.1$ & $3.5 \pm 0.2$ & $8.8 \pm 0.4$ & $5.1 \pm 0.2$ & $11.0 \pm 0.2$ & $23.8 \pm 0.5$ & $11 \pm 2$ & $21 \pm 2$ & $73 \pm 2$ & $23 \pm 2$ & $3.6 \pm 0.2$ \\
\hline & 14 days & $3.0 \pm 0.2$ & $3.4 \pm 0.1$ & $3.3 \pm 0.1$ & $8.3 \pm 0.2$ & $4.9 \pm 0.1$ & $11.0 \pm 0.2$ & $24.2 \pm 0.4$ & $13 \pm 2$ & $21 \pm 2$ & $73 \pm 2$ & $23 \pm 2$ & $3.7 \pm 0.1$ \\
\hline & $p$-Value $(n=27)$ & $<0.001$ & $<0.001$ & $<0.001$ & $<0.001$ & $<0.001$ & $<0.001$ & $<0.001$ & 0.005 & 0.234 & 0.155 & 0.201 & 0.004 \\
\hline \multirow[t]{4}{*}{ FT } & None & $3.5 \pm 0.4$ & $3.5 \pm 0.1$ & $3.3 \pm 0.1$ & $8.3 \pm 0.3$ & $4.9 \pm 0.2$ & $10.9 \pm 0.3$ & $24.0 \pm 0.4$ & $14 \pm 1$ & $19 \pm 1$ & $75 \pm 1$ & $21 \pm 1$ & $3.8 \pm 0.1$ \\
\hline & Microspheres & $3.6 \pm 0.3$ & $3.5 \pm 0.1$ & $3.3 \pm 0.1$ & $8.4 \pm 0.3$ & $4.8 \pm 0.1$ & $10.9 \pm 0.2$ & $23.9 \pm 0.5$ & $11 \pm 1$ & $23 \pm 1$ & $72 \pm 1$ & $25 \pm 1$ & $3.6 \pm 0.1$ \\
\hline & Extract & $3.6 \pm 0.5$ & $3.5 \pm 0.2$ & $3.5 \pm 0.2$ & $8.6 \pm 0.4$ & $5.0 \pm 0.2$ & $10.9 \pm 0.3$ & $23.8 \pm 0.3$ & $12 \pm 2$ & $21 \pm 1$ & $73 \pm 1$ & $24 \pm 1$ & $3.6 \pm 0.2$ \\
\hline & $p$-Value $(n=27)$ & 0.951 & 0.452 & 0.001 & 0.006 & 0.062 & 0.553 & 0.196 & $<0.001$ & $<0.001$ & $<0.001$ & $<0.001$ & $<0.001$ \\
\hline $\mathrm{ST} \times \mathrm{FT}$ & $p$-Value $(n=81)$ & $<0.001$ & $<0.001$ & 0.001 & 0.022 & $<0.001$ & $<0.001$ & $<0.001$ & $<0.001$ & $<0.001$ & $<0.001$ & $<0.001$ & 0.005 \\
\hline
\end{tabular}
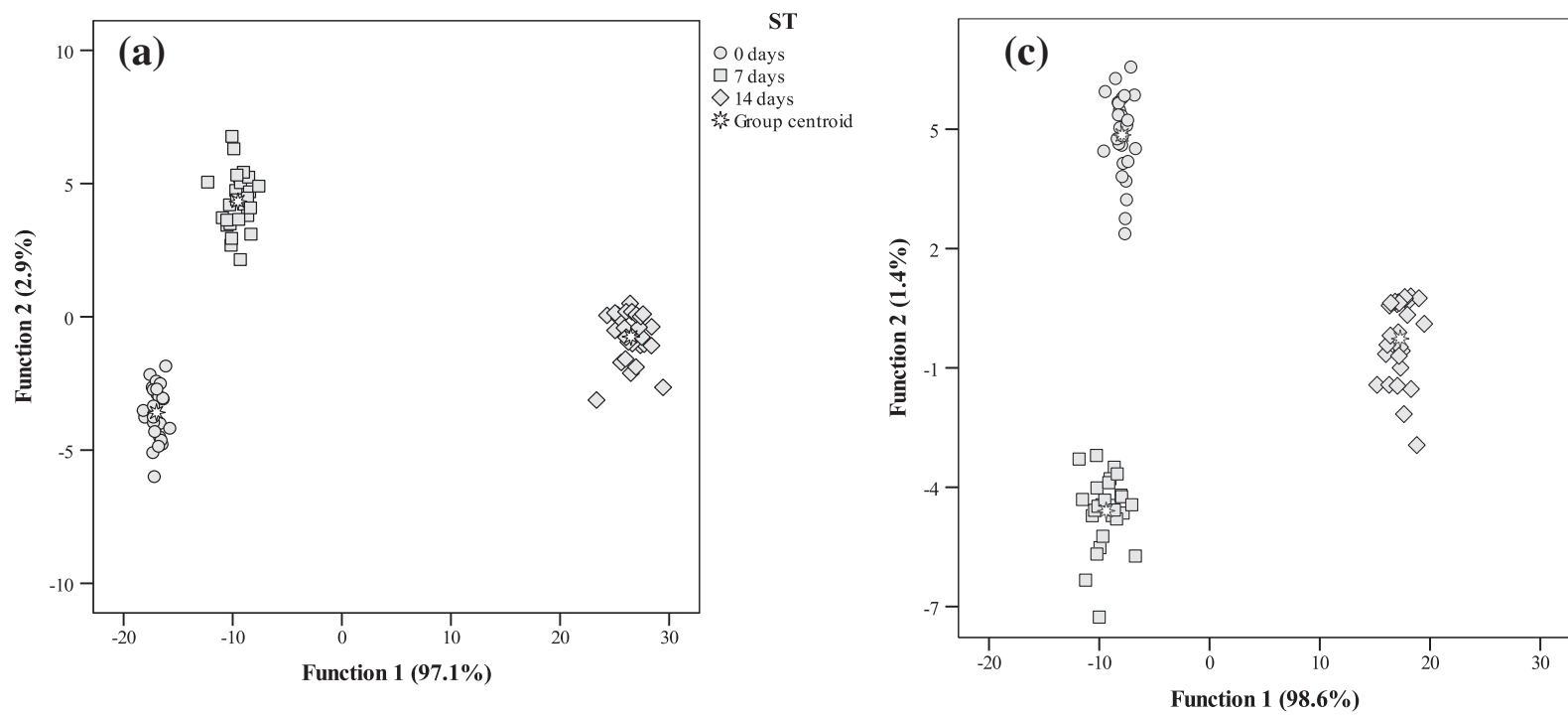

ST
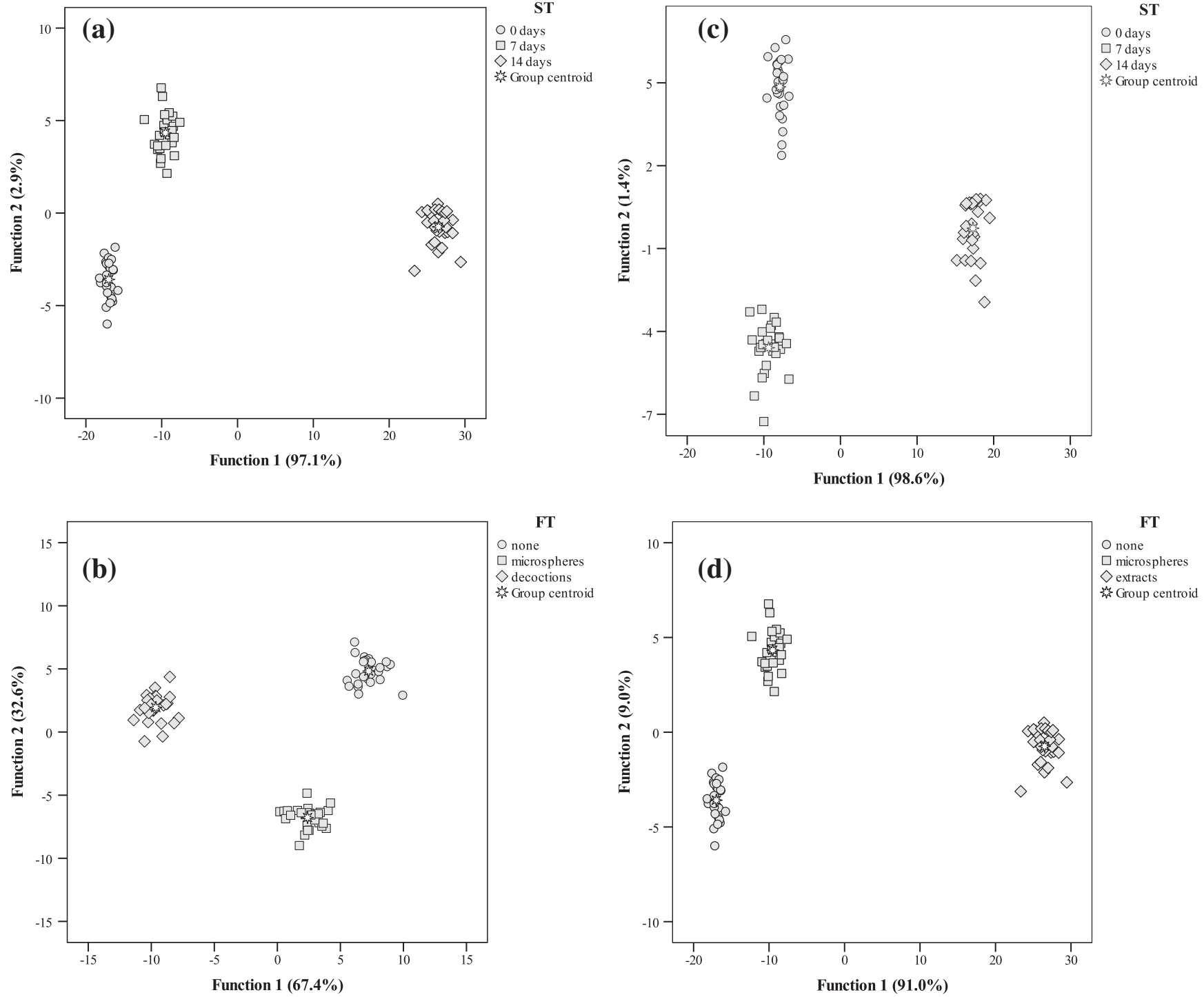

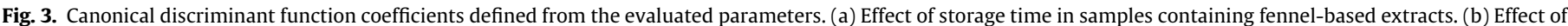

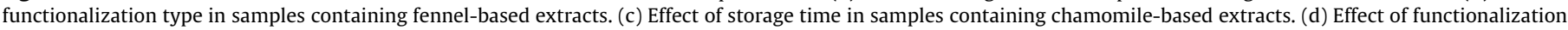
type in samples containing chamomile-based extracts. 
main differences were related to $\mathrm{C} 12: 0, \mathrm{C} 10: 0$ (higher in "7 days" samples), which were shown to be the variables with the highest correlation with function 2 .

Regarding the effect of FT, the separation among markers (Fig. 3d) reveals that the most marked differences between plain cottage cheese and samples functionalized by directly adding decocted extracts are related to function 1 , which was verified to have the strongest correlations with DPPH scavenging activity and carbohydrates. On the other hand, samples functionalized with microencapsulated extracts distinguish from plain cottage cheese through function 2, which was more highly correlated with SFA, and, particularly, C18:0. The other selected variables that displayed discriminant ability were fat, protein, ash, lactose, $L^{*}$, C4:0, C6:0 and C16:0.

The classification performance was $100 \%$ accurate for all of the LDA performed, not only for original grouped cases, but for the cross-validated grouped cases.

\section{Conclusions}

Considering all of the results together, it can be concluded that ST exerted the main effect on the nutritional composition of cottage cheese, as the variables most related to function 1 (that include most of the variance in both cases) are related to the nutritional parameters. Likewise, it is possible to identify DPPH scavenging activity as the main variable in discriminating nonfunctionalized and functionalized samples, since it was the variable most correlated with function 1 . In view of these results, it is possible to conclude that cottage cheese was effectively functionalized without causing significant changes in nutritional and color parameters, or in fatty acids profiles. Furthermore, it is also possible to deduce that microsphere encapsulation can be applied as a feasible technique to preserve antioxidant activity throughout longer storage times (Fig. 2). In fact, the microencapsulated compounds seemed to be released after the 7 th day, granting an increase in the antioxidant activity, while this bioactivity indicator started to increase for cheese samples containing free extracts after the same period.

\section{Acknowledgements}

The authors are grateful for financial support to CIMO (Project PEst-OE/AGR/UI0690/2014). Thanks are also due to FCT/MEC and FEDER under Program PT2020 for financial support to LSRE (Project UID/EQU/50020/2013). Also QREN, ON2 and FEDER (NORTE-070124-FEDER-000014) and PRODER (Project $n^{\circ}$ 46577- PlantLact) and to the Foundation for Science and Technology (FCT, Portugal) for C. Caleja's PhD Grant (SFRH/BD/93007/2013), João C.M. Barreira's Post-Doc Grant (SFRH/BPD/72802/2010) and L. Barros's research contract. The authors also thank Ms Clarinda Paixão, from Américo Duarte Paixão Lda, for providing the plant samples and Queijos Casa Matias, for providing the cottage cheese samples.

\section{References}

AOAC (2005). Official methods of analysis of AOAC international. In W. Horwitz \& G. Latimer (Eds.). Gaithersburg, MD: AOAC International (18th ed.).

Barros, L., Heleno, S., Carvalho, A., \& Ferreira, I. C. F. R. (2009). Systematic evaluation of the antioxidant potential of different parts of Foeniculum vulgare Mill. from Portugal. Food and Chemical Toxicology, 47, 2458-2464.
Barros, L., Pereira, E., Calhelha, R. C., Dueñas, M., Carvalho, A. M., Santos-Buelga, C., \& Ferreira, I. C. F. R. (2013). Bioactivity and chemical characterization in hydrophilic and lipophilic compounds of Chenopodium ambrosioides L. Journal of Functional Foods, 5, 1732-1740.

Betz, M., \& Kulozik, U. (2011). Microencapsulation of bioactive bilberry anthocyanins by means of whey protein gels. Procedia Food Science, 1, 2046-2056

Buffa, M., Trujillo, A. J., Pavia, M., \& Guamis, B. (2001). Changes in textural, microstructural, and colour characteristics during ripening of cheeses made from raw, pasteurized or highpressure-treated goats' milk. International Dairy Journal, 11, 927-934.

Caleja, C., Barros, L., Antonio, A. L., Ciric, A., Barreira, J. C. M., Soković, M.,... Ferreira, I C. F. R. (2015). Development of a functional dairy food: exploring bioactive and preservation effects of chamomile (Matricaria recutita L.). Journal of Functional Foods, 16, 114-124

Caleja, C., Barros, L., Antonio, A. L., Ciric, A., Soković, M., Oliveira, M. B. P. P., .. Ferreira, I. C. F. R. (2015). Foeniculum vulgare Mill. as natural conservation enhancer and health promoter by incorporation in cottage cheese. Journal of Functional Foods, 12, 428-438.

Çam, M., Içyer, N. C., \& Erdogan, F. (2014). Pomegranate peel phenolics: Microencapsulation, storage stability and potential ingredient for functional food development. LWT - Food Science and Technology, 55, 117-123.

Carocho, M., Barreiro, M., Morales, P., \& Ferreira, I. C. F. R. (2014). Adding molecules to food, pros and cons: A review on synthetic and natural food additives. Comprehensive Reviews in Food Science and Food Safety, 13, 377-399.

Dias, M. I., Barros, L., Fernandes, I. P., Ruphuy, G., Oliveira, M. B. P. P., Santos-Buelga, C., ... Ferreira, I. C. F. R. (2015). A bioactive formulation based on Fragaria vesca L. vegetative parts: Chemical characterization and application in $\kappa$-carrageenan gelatin. Journal of Functional Foods, 16, 243-255.

Dias, M. I., Ferreira, I. C. F. R., \& Barreiro, M. F. (2015). Microencapsulation of bioactives for food applications. Food E Function. http://dx.doi.org/10.1039/ c4fo01175a.

Diezhandino, I., Fernández, D., González, L., McSweeney, P. L. H., \& Fresno, J. M (2015). Microbiological, physico-chemical and proteolytic changes in a Spanish blue cheese during ripening (Valdeón cheese). Food Chemistry, 168, 134-141.

Farahani, G., Ezzatpanah, H., \& Abbasi, S. (2014). Characterization of Siahmazg cheese, an Iranian ewe's milk variety: Assessment of physico-chemical, textural and rheological specifications during ripening. LWT - Food Science and Technology, 58, 335-342.

Fernandes, Â., Antonio, A. L., Barreira, J. C. M., Oliveira, M. B. P. P., Martins, A., \& Ferreira, I. C. F. R. (2012). Effects of gamma irradiation on physical parameters of Lactarius deliciosus wild edible mushrooms. Postharvest Biology and Technology, $74,79-84$.

George, M., \& Abraham, T. E. (2006). Polyionic hydrocolloids for the intestinal delivery of protein drugs: Alginate and chitosan - A review. Journal of Controlled Release, 114, 1-14.

Ginzinger, W., Jaros, D., Lavanchy, P. \& Rohm, H. (1999). Raw milk flora affects composition and quality of Bergkäse-3. Physical and sensory properties, and conclusions. Le Lait-Dairy Science and Technology, 79, 411-421.

Goh, C. H., Heng, P. W. S., \& Chan, L. W. (2012). Alginates as a useful natural polymer for microencapsulation and therapeutic applications. Carbohydrate Polymers, 88 $1-12$.

Guimarães, R., Barros, L., Dueñas, M., Calhelha, R. C., Carvalho, A. M., Santos-Buelga, C. ... Ferreira, I. C. F. R. (2013). Infusion and decoction of wild German chamomile: Bioactivity and characterization of organic acids and phenolic compounds. Food Chemistry, 136, 947-954.

Martins, A., Barros, L., Carvalho, A. M., Santos-Buelga, C., Fernandes, I. P., Barreiro, F. \& Ferreira, I. C. F. R. (2014). Phenolic extracts of Rubus ulmifolius Schott flowers: Characterization, microencapsulation and incorporation into yogurts as nutraceutical sources. Food E' Function, 5, 1091-1100.

Mata, A. T., Proença, C., Ferreira, A. R., Serralheiro, M. L. M., Nogueira, J. M. F., \& Araújo, M. E. M. (2007). Antioxidant and antiacetylcholinesterase activities of five plants used as Portuguese food spices. Food Chemistry, 103, 778-786.

Pizzillo, M., Claps, S., Cifuni, G., Fedele, V., \& Rubino, R. (2005). Effect of goat breed on the sensory, chemical and nutritional characteristics of ricotta cheese. Livestock Production Science, 94, 33-40.

Queiroga, R., Santos, B., Gomes, A., Monteiro, M., Teixeira, S., Souza, E., ... Pintado, M. (2013). Nutritional, textural and sensory properties of Coalho cheese made of goats', cows' milk and their mixture. LWT - Food Science and Technology, 50, 538-544.

Rohm, H., \& Jaros, D. (1996). Colour of hardcheese-1. Description of colour properties and effects of maturation. Zeitschrift fur Lebensmittel Untersuchung Forschung, 203, 241-244.

Zielinski, A. A. F., Haminiuk, C. W. I., Alberti, A., Nogueira, A., Demiate, I. M., \& Granato, D. (2014). A comparative study of the phenolic compounds and the in vitro antioxidant activity of different Brazilian teas using multivariate statistical techniques. Food Research International, 60, 246-254. 